\title{
Hinode SOT/SP and SoHO/MDI quiet Sun magnetic field. Implications of their differences on the extrapolated chromospheric field and the height of the magnetic canopy
}

\author{
I. Kontogiannis ${ }^{1,2}$, G. Tsiropoula ${ }^{1}$, and K. Tziotziou ${ }^{1}$
}

\begin{abstract}
${ }^{1}$ National Observatory of Athens, Institute for Space Applications and Remote Sensing, Lofos Koufos, 15236 Palea Penteli, Greece e-mail: [jkonto; georgia; kostas] @space.noa.gr

2 Department of Astrophysics, Astronomy and Mechanics, Faculty of Physics, National and Kapodistrian University of Athens, 15784 Zografos, Greece
\end{abstract}

Received 22 February 2011 / Accepted 20 May 2011

\begin{abstract}
Aims. We explore the differences in the measurement of the magnetic field of the quiet solar photosphere provided by the Michelson Doppler Imager (MDI) onboard SoHO and the SpectroPolarimeter (SOT/SP) onboard Hinode and the ensuing implications for the extrapolated chromospheric magnetic field and the determination of the location of the magnetic canopy.

Methods. We employ potential field extrapolation to reconstruct the chromospheric magnetic field using the magnetic field of the photosphere provided by the two instruments. We also calculate the plasma- $\beta$ parameter using the VAL C model atmosphere of the quiet Sun to determine the height of the magnetic canopy.

Results. MDI underestimates the magnetic field of the quiet Sun sometimes by a factor of five, which leads to an overestimation of the height of the magnetic canopy by up to $\sim 550 \mathrm{~km}$. Although the overall magnetic field configuration does not differ significantly when calculated with either MDI or SOT/SP, the data of the latter lead to lower and more extended canopies. The difference in the resolution of the two instruments does not seem to affect the chromospheric magnetic field higher than $1000 \mathrm{~km}$.

Conclusions. The height of the magnetic canopy is an important parameter to consider when investigating wave propagation and the oscillatory properties of the quiet Sun regions in the network and internetwork. The canopy height's derivation depends very much on the sensitivity of the instruments used to measure the photospheric magnetic field. Consequently precise measurements of the photospheric magnetic field are crucial to accurately reconstruct the chromospheric magnetic field and to distinguish between the various wave modes.
\end{abstract}

Key words. Sun: chromosphere - Sun: photosphere - Sun: surface magnetism

\section{Introduction}

The magnetic field of the quiet Sun is, usually, organized in a cellular pattern that is known as the magnetic network. The magnetic network is formed by the magnetic flux transported to the boundaries of supergranular cells by the convective flows and is made up from clusters of magnetic elements called network bright points (NBPs). These are the visible manifestation of up to $\mathrm{kG}$-strength flux tubes. As these flux tubes fan out with height because of the exponential drop of the gas pressure, they give rise to interesting phenomena, such as the formation of the magnetic canopy, and have important implications in the propagation of waves.

The role of the magnetic field of the quiet Sun in the propagation of waves has been explored through a number of observational and theoretical studies. So far, in terms of wave propagation in the solar atmosphere, two components have been considered: the magnetized network boundaries and the internetwork (IN), which is devoid of intense magnetic flux. The IN is usually considered "field free" for the purposes of these studies, although increasingly higher-resolution observations reveal the opposite (Lites et al. 2008). The different character of the oscillations and waves has been pointed out by several authors (Lites et al. 1982; Lites et al. 1993; Krijger et al. 2001). Investigations of the spatial distribution of the oscillatory power have revealed areas called "power halos" where the power is enhanced, and areas called "magnetic shadows" where the power is suppressed. Power halos were first observed in active regions at the photosphere around the plages (Braun et al. 1992; Brown et al. 1992; Hindman \& Braun 1998; Thomas \& Stanchfield 2000; Jain \& Haber 2002; Muglach 2003). It was also found that photospheric p-modes are suppressed over these extended magnetic concentrations. Judge et al. (2001) found power suppression over the chromospheric network and several studies have confirmed this finding (McIntosh \& Judge 2001; McIntosh et al. 2003; Vecchio et al. 2007; Kontogiannis et al. 2010a).

The interaction of magneto-acoustic waves with the magnetic canopy has been put forth to explain these power deficits and enhancements (McIntosh et al. 2003; Muglach et al. 2005; Kontogiannis et al. 2010a, b, hereafter Paper I), because it is on this critical surface that waves undergo refraction, reflection, and mode conversion (Rosenthal et al. 2002; Bogdan et al. 2003). An important conclusion drawn from these studies is that it is imperative to know the location of the canopy relative to the formation height of the bandpass one uses to observe before deciding which kind of waves and interactions may produce the observed phenomena. Furthermore, acoustic waves carry energy which, in the presence of a fully magnetized chromosphere, should be channeled to the upper solar atmosphere in the form of magnetoacoustic waves, making them a possible candidate for heating 
(Jefferies et al. 2006). These remarks make clear that the precise knowledge of the quiet Sun magnetic field and of the location of the magnetic canopy are very important ingredients in a realistic model of wave (energy) propagation.

Because the canopy is defined by the equilibrium between magnetic and gas pressure, the determination of its location depends on two factors: the precise measurement of the photospheric magnetic fields and the height profile of the variation of the thermodynamical parameters (i.e., an accurate solar model). An array of studies has been dedicated to the comparison between instruments and spectral lines used for the determination of the photospheric magnetic field. The purpose is to assess whether certain spectral lines or instruments provide accurate measurements and are suitable for certain types of studies. For instance, the comparison of the Michelson Doppler Imager (MDI) onboard the Solar Heliospheric Observatory (SoHO) with other instruments has several times led to the re-calibration of its data (see Wang et al. 2009 for a short review of this topic). For this type of studies a large sample of areas, that are distributed over the entire solar disk is needed (such as in Demidov \& Balthasar 2009; Wang et al. 2009; and Ulrich et al. 2009) to establish a calibration relation as a function of the observation angle.

Motivated by the importance of the magnetic canopy on wave propagation, we aim to demonstrate how using different inputs for determining the chromospheric magnetic field through current-free magnetic field extrapolations may affect the derived conclusions. We examine and highlight the consequences of using magnetograms of a solar quiet region observed by two instruments with different resolutions and sensitivities, i.e., MDI onboard SoHO and the SpectroPolarimeter (SP) of the Solar Optical Telescope (SOT) onboard Hinode (SOT/SP), in the determination of the location of the magnetic canopy.

\section{Observations}

The observations used in this work were obtained on October 15, 2007, during a coordinated campaign, which included several ground-based and space instruments. Filtergrams, spectrograms, and magnetograms of a solar quiet region located at the solar disk center were taken. For the magnetograms, both MDI/SoHO and SOT/SP onboard the Hinode mission were used.

MDI (Scherrer et al. 1995) on-board SoHO provides high-resolution magnetograms as well as photospheric Doppler velocity maps and continuum intensity images. Instead of scanning the whole profile, MDI produces filtergrams in certain polarization states and wavelengths around the Ni I ( $\lambda 6767.8 \AA$ ) line. The produced filtergrams are then calibrated to provide the line-of-sight (LOS) magnetic field, intensity, and velocity at the height-of-formation (HOF) of the Ni I line $(\sim 200 \mathrm{~km})$. Through the operation years of MDI, several re-calibrations have been performed, based on the comparison of MDI with other more accurate instruments (Wang et al. 2009), which eventually lead to the most recent level-1.8 data,which were used in this work. The quiet solar area was observed by MDI between 8:00-11:00 UT. The pixel size of the high-resolution MDI images is $0.6^{\prime \prime}$ and their cadence is $60 \mathrm{~s}$.

SOT/SP performed two raster scans in the Fe I lines at 6301.5 $\AA$ and 6302.5 $\AA$ taken between 9:05-9:15 UT and 9:159:25 UT. SOT/SP operated in the fast-map mode with an effective pixel size of 0 ' 32 , covering a $50^{\prime \prime} \times 164^{\prime \prime}$ field-of-view (FOV) in $10 \mathrm{~min}$. The HAO/CSAC team performed the inversion of the Stokes spectra using the MERLIN code, which is based on the Milne-Eddington (ME) approximation. According to this approach, the source function varies linearly along the LOS, while the magnetic field vector, magnetic filling factor, line strength, Doppler shift, and line broadening are kept constant. Then the observed line profiles were least-squares fitted using a magnetized component and a scattered-light profile. The output of this procedure is the magnitude of the magnetic field along with its inclination, azimuth, filling factor, and several other parameters. The reader may refer to the HAO/CSAC team website ${ }^{1}$ for further information concerning the inversion procedure.

The use of ME inversion is an approximation that allows a relatively fast processing of large sets of data. Its accuracy and reliability, however, are constantly monitored. On the one hand, the fitting procedure is robust, but for weak fields it may not converge (Lites et al. 2008). Using a threshold in the polarization signal and rejecting the pixels with weak signals is a common strategy (Orozco Suárez et al. 2007; Jin et al. 2009) to ensure the convergence of the fit. In our case all NBPs that are of concern here as well as strong IN magnetic elements are several times above the noise level, which means that the fit there is acceptable. On the other hand, the simplifying assumptions of the ME approximation (no gradients of the parameters along the LOS) often raise questions about the validity of the results. Although this is a topic that is constantly under examination, it is generally accepted that the Milne-Eddington approximation gives fair estimates over the range of formation of the line used (Westendorp Plaza et al. 1998) and this is not only the case at active regions, but also at finer magnetic concentrations, as has been demonstrated by Orozco Suárez et al. (2010). In this study, a crosscomparison with MHD simulations has shown that the magnetic field flux for the Fe I lines closely matches the simulated flux at $\log \tau=-1$. Assuming the VAL C model (Vernazza et al. 1981), this height is no more than $\sim 100 \mathrm{~km}$ lower than the HOF of the $\mathrm{Fe}$ I line, and given the corresponding pressure stratification, it does not affect our results.

The vertical component of the photospheric magnetic field is $B_{\mathrm{LOS}}=B_{0} \alpha \cos \psi$, where $\alpha$ is the filling factor, $B_{0}$ the magnitude of the magnetic field and $\psi$ the inclination of the magnetic field vector to the LOS. Because the area under study is located at the solar disk center and the common FOV (which is the SOT/SP's FOV) has a limited horizontal spatial extent, no corrections on the magnetic field vector were applied assuming that the LOS is the local vertical. Therefore, the vertical component of the magnetic field is the LOS component and the transverse one is the horizontal. The HOF of the Fe I lines used by SOT/SP is at $\sim 250 \mathrm{~km}$ (Shchukina \& Trujillo Buenno 2001) very close to the HOF of Ni I. In the following, all heights will be measured not from the HOF of either of the two lines, but from $\tau_{5000}=1$.

\section{Data analysis}

The MDI and SOT/SP LOS magnetograms were co-aligned by means of cross-correlation and then the common FOV was extracted. The SOT/SP magnetograms were also degraded to the spatial scale of MDI, that is $0.6^{\prime \prime}$ per pixel. Out of the MDI series of frames a synthetic raster was created by dividing the SOT/SP FOV in eleven 1-minute stripes and selecting the corresponding stripes of the co-temporal MDI frames. In order to increase the signal-to-noise ratio of the MDI magnetograms an average MDI frame was also calculated using the 11 frames that were taken during the SOT/SP raster acquisition. In Fig. 1 the first SOT/SP

1 http://www.csac.hao. ucar.edu/csac/dataHostSearch.jsp 

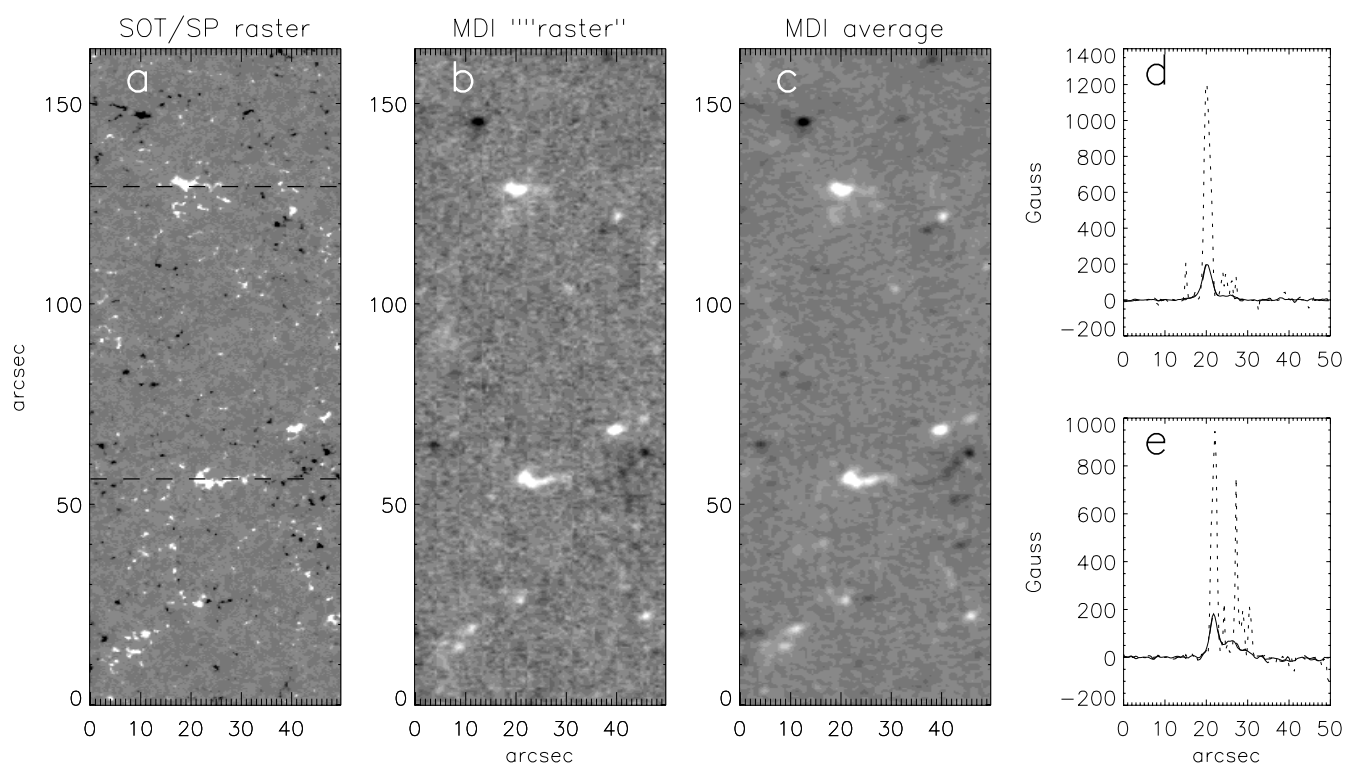

Fig. 1. a) SOT/SP raster taken between 9:05-9:15 UT. b) Co-temporal MDI synthetic raster. c) MDI average magnetogram obtained from the co-temporal to the SOT/SP frames MDI magnetograms. d) LOS magnetic field along the upper dashed line indicated in panel a) for the SOT/SP (dotted line), MDI average and synthetic raster (solid and dashed lines). The corresponding values of the MDI synthetic raster are almost identical to the ones from the average frame. e) Same as d) but for the lower dashed line in panel a).

raster (used here) is presented along with the corresponding MDI synthetic raster and MDI average frame.

For further analysis, a subregion of the FOV was selected. This is the same region as the one studied in Paper I, which contains a clearly defined part of the network and ambient IN. The three-dimensional chromospheric magnetic configuration was reconstructed using the current-free (potential) field approximation (Schmidt 1964). This type of extrapolation requires the LOS magnetic field of the lower boundary, that is, the photosphere or the HOF of the spectral line used $(250 \mathrm{~km}$ for the SOT/SP and $200 \mathrm{~km}$ for the MDI), and uses Green's functions to provide the vector of the magnetic field at equidistant heights in the chromosphere. The height resolution is equal to the spatial resolution of the input magnetogram. More details on the procedure can be found in Paper I. The extrapolation was performed using four different inputs: the MDI raster and average magnetograms, the SOT/SP magnetogram in MDI resolution and the SOT/SP magnetogram in its full resolution. As already noted, the LOS component of the extrapolated magnetic field is the magnetic field along the $z$-axis, while the transverse (TRANS) component is $B_{\mathrm{TRANS}}=\sqrt{B_{x}^{2}+B_{y}^{2}}$, where $B_{x}$ and $B_{y}$ are the components of the magnetic field along the $x$ and $y$-axis, respectively. The inclination of the magnetic field to the LOS is $\theta=\arctan \left(B_{\text {TRANS }} / B_{\text {LOS }}\right)$.

With the extrapolated chromospheric field at hand, we calculated the plasma- $\beta$ parameter, i.e., the ratio between the gas pressure versus the magnetic pressure $\left(P_{\mathrm{mag}}=B^{2} / 2 \mu_{0}\right)$. The gas pressure was taken from the model $\mathrm{C}$ of the average quiet Sun of Vernazza et al. (1981) and the pressure at each height of the extrapolated magnetic field was determined by means of spline interpolation. The heights at which $\beta$ is on the order of unity were also determined, i.e., the heights where gas and magnetic field are in pressure equilibrium. We call these the $\beta$ transition heights, $\beta_{\mathrm{TH}}$ (McIntosh et al. 2003) or canopy heights because they represent the height of the magnetic canopy. The azimuthally averaged profiles of the LOS, TRANS components, inclination of the magnetic field, and $\beta$ are calculated over concentric circles around a certain spot on the network (see Paper I) located at $\sim(X, Y)=\left(20^{\prime \prime}, 58^{\prime \prime}\right)$ (Fig. 1) and this was made for every height of the extrapolated magnetic field. These profiles give a measure of the variation of the above parameters as a function of distance from the network.

\section{Results}

\subsection{SOT/SP and MDI magnetic fluxes of the quiet Sun}

Figure 1 shows a quiet Sun area, located at disk center (which is at $\sim\left(X_{\mathrm{CEN}}, Y_{\mathrm{CEN}}\right)=\left(20^{\prime \prime}, 65^{\prime \prime}\right)$ as observed by SOT/SP. The area contains three NBP clusters of positive polarity located at $\sim(X, Y)=\left(20^{\prime \prime}, 55^{\prime \prime}\right),\left(35^{\prime \prime}, 68^{\prime \prime}\right)$ and $\left(18^{\prime \prime}, 129^{\prime \prime}\right)$, as well as several IN magnetic elements of mixed polarities. The majority of these is lost within the noisy pattern of the IN at the MDI synthetic raster. Remarkably, even the clearly defined network appears blurry at the MDI resolution. In the MDI average frame (panel c), on the other hand, several IN magnetic elements and the network appear in better contrast.

In panels $d$ and e of Fig. 1 we plot the values of the LOS magnetic field along the horizontal lines (dashed lines in panel a) that cross two NBP clusters at $(X, Y)=\left(20^{\prime \prime}, 56^{\prime \prime}\right)$ and $(X, Y)$ $=\left(18^{\prime \prime}, 129^{\prime \prime}\right)$ in the SOT/SP raster and the average MDI frame. Note that the values of the magnetic field of the NBP cluster are more than four times lower in MDI compared to those given by SOT/SP. The values of the MDI synthetic raster and average frame almost coincide, regardless of the different contrast.

That MDI underestimates the magnetic flux density compared to that of SOT/SP has already been reported for active regions. Moon et al. (2007) have found lower MDI (operating at the high-resolution mode) flux densities by a factor of two at penumbral regions compared to that of SOT/SP (operating in normal mode). A similar result was found by Wang et al. (2009), who used a sample of 45 active regions, but compared SOT/SP with MDI full disk low-resolution magnetograms and found that 

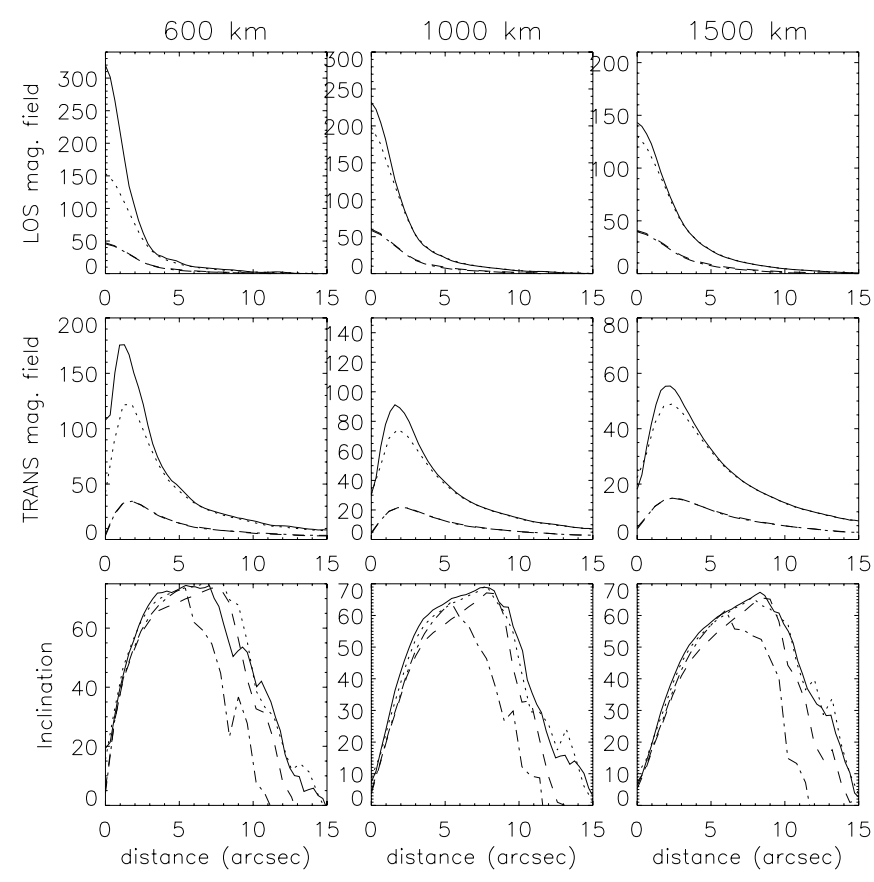

Fig. 2. Azimuthally averaged profiles of the LOS and TRANS components and the inclination to the LOS of the extrapolated magnetic field as a function of the distance from a location taken at the center of the network region. The profiles are calculated at 600, 1000, and $1500 \mathrm{~km}$ above the photosphere from the MDI average frame (dashed line) and raster (dashed-dotted), SOT/SP in MDI resolution (dotted line) and SOT/SP in full resolution (solid line) magnetograms.

MDI values are 70-80\% that of SOT/SP. That this difference appears to be larger in the quiet Sun is somehow anticipated and is mostly attributed to the difference in spatial resolution of the two instruments. The resolution of MDI is almost twice that of the SOT/SP, and as a result the filling factor for an MDI pixel is much lower than that of the SOT/SP, which will lead to the calculation of a lower magnetic flux by MDI. This effect is enhanced at the quiet Sun where the magnetic distributions are not extended. It should be noted that the different lines used by the two instruments to measure the magnetic field should not affect the measured magnetic field so drastically. The HOF of the two lines are separated by $\sim 50 \mathrm{~km}$, a distance over which the variation of the magnetic field is insignificant (Berger \& Lites 2003).

The differences in the measured magnetic fields in the photosphere propagate also, as expected, to the extrapolated magnetic field at the chromosphere. The variation of the LOS and TRANS components and of the inclination to the LOS of the magnetic field as a function of distance from the network is shown in Fig. 2 for three different heights. The heights chosen (measured from $\tau_{5000}=1$ ) are: one at $600 \mathrm{~km}$, which is near the temperature minimum, one at $1000 \mathrm{~km}$ and one at $1500 \mathrm{~km}$. An extensive description and comments on the shape of the curves and their variation with height can be found in Paper I.

Both MDI raster and average image produce almost identical chromospheric potential magnetic fields. As can be seen in Fig. 2, the dashed and dashed-dotted lines, which correspond to the two different MDI inputs, almost coincide at all heights for both LOS and TRANS components. We now focus on the differences between the SOT/SP and MDI extrapolated magnetic field. At all heights, both LOS and TRANS components have lower values when calculated from the MDI (dashed line) than those calculated using SOT/SP, in MDI (dotted line) and full
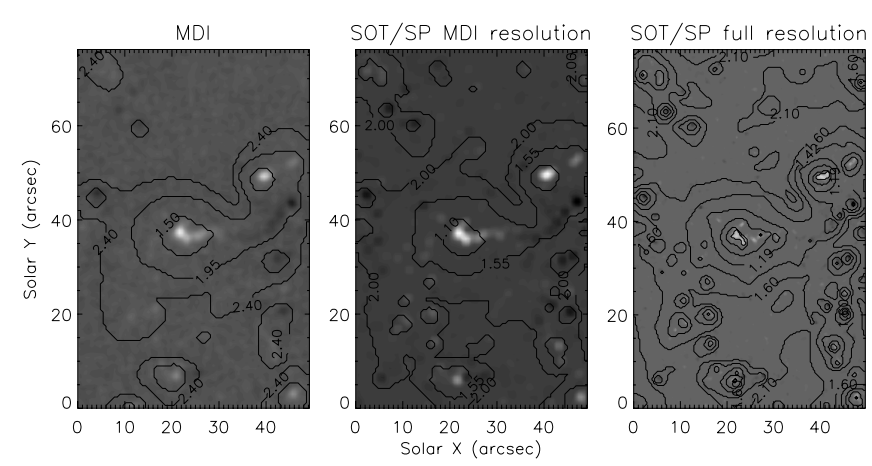

Fig. 3. MDI, SOT/SP in MDI resolution and SOT/SP in full resolution magnetograms with contours of the canopy heights overplotted.

resolution (solid line). A visual inspection shows that the ratio between MDI and SOT/SP values near the NBPs decreases as the height increases. The difference between the two SOT/SP curves is very small and at $1500 \mathrm{~km}$ they almost coincide. The values of the photospheric magnetic field of the network affect the chromospheric magnetic field more than does the resolution of the input SOT/SP magnetogram. This is perhaps an indication that the fine structure of the photospheric magnetic network and IN does not affect the chromospheric magnetic configuration at or above $1500 \mathrm{~km}$, when a potential magnetic field is assumed for the extrapolation.

Apart from the differences in the LOS and TRANS components, the inclination values do not differ significantly. The corresponding curves in the third row of Fig. 2 are almost identical for all four cases, but the inclination profile of the MDI raster is noisier. This means that the overall configuration of the magnetic field is almost the same whether we use MDI or SOT/SP magnetograms as an input.

\subsection{The height and shape of the magnetic canopy}

MDI, SOT/SP at MDI resolution and SOT/SP at full resolution images of the photospheric longitudinal magnetic field of a subregion of Fig. 1 showing part of the magnetic network are given in Fig. 3. The overplotted contours represent canopy heights (or $\beta_{\mathrm{TH}}$ ) calculated from the extrapolated potential magnetic field and the average quiet Sun model atmosphere (model C) of Vernazza et al. (1981). In this figure we present the canopies of the chromospheric potential magnetic field of the average MDI frame (left panel). We obtained identical results with the synthetic raster. The overall appearance of the contours, i.e., the "shape" of the canopy, is similar in MDI and SOT/SP. The extrapolated potential field would have the same general configuration as the one shown in Fig. 3 of Paper I. There is, however, a significant difference in the values of the canopy heights. Note that the $1.95 \mathrm{Mm}$ contour in the left panel of Fig. 3 corresponds to the $1.6 \mathrm{Mm}$ contour in the middle panel of Fig. 3. At that distance from the network, the lower MDI magnetic field values result in a $\beta_{\mathrm{TH}}$ situated about $350 \mathrm{~km}$ higher than the one calculated using the SOT/SP magnetograms. In the right panel of Fig. 3, where the full SOT/SP resolution is used, the shape of the canopy is more detailed with more contours packed closer. Furthermore, the higher resolution of the corresponding input magnetogram leads to a canopy situated even lower. Note that the $1.95 \mathrm{Mm}$ contour of the left panel corresponds roughly to the $1.4 \mathrm{Mm}$ contour of the right panel, a difference of $\sim 550 \mathrm{~km}$. In Paper I we showed that the latter representation 

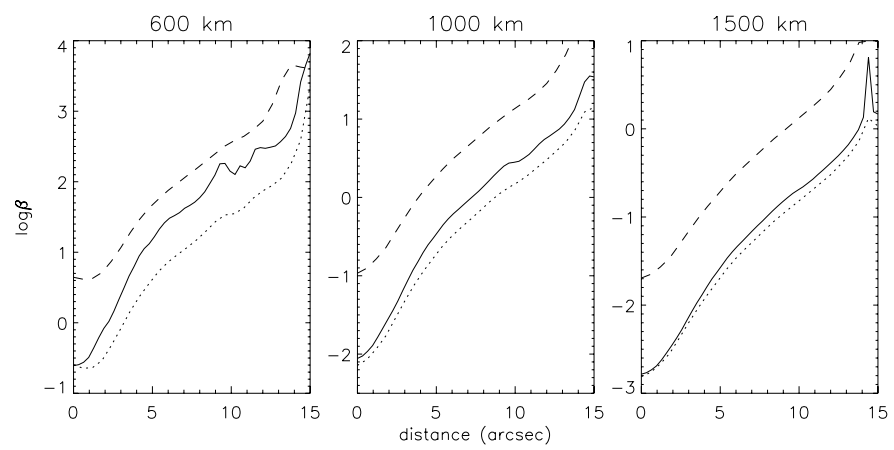

Fig. 4. Same as Fig. 2, but for the logarithm of the plasma- $\beta$.

of the magnetic canopy agrees remarkably well with the view provided by the $\mathrm{H} \alpha$ Doppler signal power maps of 3 and 5 min oscillations (Fig. 4 of Paper I).

Furthermore, the $\beta_{\mathrm{TH}}$ is also significantly modified over the IN when using the high-resolution SOT/SP magnetogram. As can be seen in the left panel of Fig. 3, the MDI IN contains almost no magnetic elements and the canopy is located above $2.4 \mathrm{Mm}$. However, the presence of IN magnetic field concentrations in the SOT/SP magnetograms results in lower $\beta_{\mathrm{TH}}$ at locations around these magnetic fields. This is an important result because the IN magnetism seems to affect the development and observation of acoustic shocks in chromospheric lines (Vecchio et al. 2009). Low-lying canopies may locally affect wave propagation even in the IN and the "hidden flux" revealed by the most recent high-resolution observations could play a significant part in channeling magnetoacoustic waves in the upper chromosphere.

The variation of plasma- $\beta$ as a function of distance from the network and its dependence on the different resolution and magnetic field values is demonstrated by the azimuthally averaged $\beta$ profiles (Fig. 4). As with the canopy heights, the calculated $\beta$ profiles for both the MDI synthetic raster and average frame are identical. The two SOT/SP curves (solid and dotted lines), albeit calculated for different resolutions, are almost identical at 1000 and $1500 \mathrm{~km}$ (middle and right panels, in Fig. 4). They differ the most at $600 \mathrm{~km}$ (Fig. 4, left panel), for distances greater than $2^{\prime \prime}$ from the NBP cluster, where $\beta$ calculated from the lowresolution SOT/SP magnetogram is lower. In general, degrading the SOT/SP resolution results in an overall smoother curve that resembles the MDI curve, only shifted toward lower $\beta$. The MDI curve is shifted toward higher $\beta$ at all heights. It is noteworthy that at $600 \mathrm{~km}, \mathrm{MDI} \log \beta$ is positive $(\beta>1)$ even above the network. This is not the case for the other two considered heights, because $\log \beta$ is negative $(\beta<1)$ above the network but still, the distance at which $\beta=1$, which is equivalent to the horizontal extent of the canopy at this height, is drastically affected. At $1000 \mathrm{~km}$ the MDI $\log \beta$ curve crosses zero at half the distance as that of the corresponding SOT/SP curves do, while at $1500 \mathrm{~km}$ the latter hardly reaches zero even at $10^{\prime \prime}$ from the network boundaries. Thus, $\beta$ values obtained from SOT/SP observations in both resolutions show that at $1500 \mathrm{~km}$ the chromosphere is almost entirely magnetically dominated, a conclusion not drawn from the MDI extrapolated magnetic field. Obviously, because the values of the magnetic field of the quiet photosphere are underestimated by the MDI, the plasma- $\beta$ parameter is overestimated in subsequent extrapolations. This result significantly alters our impression of the dynamic environment of the chromospheric plasma.

\section{Discussion and conclusions}

We used simultaneous magnetograms taken by MDI onboard SoHO and SOT/SP onboard Hinode. MDI operated at its highresolution mode, while SOT/SP performed raster scans at its fast map mode. We compared magnetic fields from an MDI average magnetogram, MDI synthetic raster, SOT/SP at its full resolution and SOT/SP degraded to MDI's resolution, as well as the subsequent extrapolated magnetic fields up to the chromospheric level. For the extrapolation of the magnetic field, the currentfree (potential) assumption was used and we also calculated the LOS and TRANS components, as well as inclination of the magnetic field to the LOS. Assuming the VAL C model atmosphere of the quiet Sun (Vernazza et al. 1981) we derived the plasma $\beta$. We found that MDI underestimates the magnetic field of the quiet Sun, sometimes up to five times, failing to measure the $\mathrm{kG}$ network fluxes known from the literature. This underestimation leads to lower values for the extrapolated magnetic field at the chromosphere. The common practice of averaging MDI frames to produce a magnetogram of better quality and better signalto-noise ratio only brings out more IN magnetic elements and shows the NBPs in better contrast.

Interestingly, however, the two instruments produced almost the same magnetic configuration in the chromosphere. We showed how the general shape of the canopy height contours (Fig. 3) is almost independent of the instrument or resolution. This is also revealed by the almost identical profiles of the magnetic field inclination shown in the lower panels of Fig. 2. There are also indications that the difference in resolution between the two instruments does not drastically affect the chromospheric magnetic field in both strength and geometry. This probably means that any fine structure in the photospheric magnetic field does not "survive" higher than $1000 \mathrm{~km}$ (in a minimum energy state such as the potential magnetic field). Longcope et al. (2009), who compared MDI full disk and SOT/NFI magnetograms, draw a similar conclusion. Searching for more null points, they found that the configuration of the magnetic field higher than $1.5 \mathrm{Mm}$ is not altered by higher resolution. Furthermore, a series of studies based on the MDI have shown that the potential magnetic field assumption agrees well with the magnetic configuration of the upper solar atmosphere inferred from X-ray, $\mathrm{H} \alpha$, or Ca II images (Perez-Suarez, et al. 2008; Vecchio et al. 2009; Kontogiannis et al. 2010b), although there are deviations from the potential field assumption (Woodard \& Chae 1999), especially at lower heights (Zhao et al. 2009).

The high-resolution, high-quality data provided by SOT/SP have shown that considering the IN free of magnetic field is far from reality. In several works, the magnitude and dynamics of the abundant IN magnetic concentrations have been explored (Lites et al. 2008; de Wijn et al. 2008). We showed here that their presence results in lower $\beta$ values and lower canopy over the IN. The impact of IN magnetic fields on wave propagation has not yet been made very clear through 2-D maps of oscillatory power, perhaps owing to their fine size, which is comparable to the resolution limit in most cases. Also, in most studies on wave propagation, IN is usually averaged, being taken as a uniform fieldfree area where acoustic waves dominate. Any contribution of the magnetic elements of the IN, if present and observable, will be washed out. However, the IN distribution of oscillatory power is not uniform (see, e.g., the power maps in Fig. 4 of Paper I) and it is possible that in the future, using even higher resolution observations, the role of IN magnetism in wave propagation and energy balance of the chromosphere will be better understood. 
We showed that high-precision measurements of the magnetic field of the network, such as those provided by SOT/SP, lead to a canopy horizontal extent that has twice the size of the one that results from the MDI magnetic network, at a height of $1000 \mathrm{~km}$ above the photosphere. Already, at the height of the temperature minimum the canopy extends horizontally up to $2^{\prime \prime}$ from the network boundaries, while MDI fails to provide any indication for the existence of a canopy at this height. This result can explain in a straightforward manner the power deficits detected over the network in the TRACE UV continua (Judge et al. 2001; Krijger et al. 2001), because the magnetic canopy is not merely situated lower than at the IN (McIntosh et al. 2003), but directly interferes with the HOF of these continua. The chromospheric network when observed with SOT/SP seems to be almost completely magnetized at the height of $1500 \mathrm{~km}$ from the photosphere. This should be seriously considered when interpreting oscillatory motions and assigning wave propagation using lines such as $\mathrm{H} \alpha$ or the $\mathrm{Ca}$ II infrared.

Theoretical studies on wave propagation (see, e.g., Rosenthal et al. 2002; Bogdan et al. 2003; Carlsson \& Bogdan 2006; Khomenko et al. 2008; Cally 2007) underline the role of the magnetic canopy in the channeling of magneto-acoustic waves in the upper atmosphere. In these studies, the authors point out that the result of the interaction between acoustic disturbances and the magnetic field depends on the attack angle, which for vertical propagation is indeed the inclination of the magnetic field. The interaction itself takes place at the so-called "equipartition layer", where the plasma- $\beta$ is on the order of unity, that is at the magnetic canopy. Even though the inclination of the chromospheric magnetic field was almost the same for both MDI and SOT/SP at all heights, this was not the case for the height of the canopy, because it depends on the accuracy with which the magnetic network flux is measured. It goes without saying that interpreting observational findings such as the oscillatory power distribution in network and IN and the observed phase differences (Kontogiannis et al. 2010a; Vecchio et al. 2007) requires the knowledge of the relative positions of the canopy and the bandpass of the filter or line used for the observations. This is the key element to distinguish which modes are the ones observed and which physical processes are taking place and this, in turn, requires the precise knowledge of network and IN magnetism.

Acknowledgements. The authors would like to thank the SoHO/MDI and Hinode teams for their efforts during the campaign. SoHO is a project of international cooperation between ESA and NASA. SOHO/MDI is a project of the Stanford-Lockheed Institude for Space Research. Hinode is a Japanese mission developed and launched by ISAS/JAXA, collaborating with NAOJ as a domestic partner, NASA and STFC (UK) as international partners. Scientific operation of the Hinode mission is conducted by the Hinode science team organized at ISAS/JAXA. This team mainly consists of scientists from institutes in the partner countries. Support for the post-launch operation is provided by JAXA and NAOJ (Japan), STFC (UK), NASA, ESA, and NSC (Norway). Hinode SOT/SP Inversions were conducted at NCAR under the framework of the Community Spectro-polarimetric Analysis Center (CSAC; http://www.csac.hao.ucar . edu). The authors would also like to thank Dr. Manolis Georgoulis for useful discussions.

\section{References}

Berger, T. E., \& Lites, B. W. 2003, Sol. Phys., 213, 213

Bogdan, T. J., Carlsson, M., Hansteen, V., et al. 2003, ApJ, 599, 626

Braun, D. C., Duvall, T. L., Jr., Labonte, B. J., et al. 1992, ApJ, 391, L113

Brown, T. M., Bogdan, T. J., Lites, B. W., \& Thomas, J. H. 1992, ApJ, 394, L65 Cally, P. S. 2007, Astron. Nachr., 328, 286

Carlsson, M., \& Bogdan, T. J. 2006, Philos. Trans. R. Soc. London A, 364, 395 Demidov, M. L., \& Balthasar, H. 2009, Sol. Phys., 260, 261

de Wijn, A. G., Lites, B. W., Berger, T. E., et al. 2008, ApJ, 684, 1469

Hindman, B. W., \& Brown, T. M. 1998, ApJ, 504, 1029

Jain, R., \& Haber, D. 2002, A\&A, 387, 1092

Jin, C., Wang, J., \& Zhao, M. 2009, ApJ, 690, 279

Jefferies, S. M., McIntosh, S. W., Armstrong, J. D., et al. 2006, ApJ, 648, L151

Judge, P. G., Tarbell, T. D., \& Wilhelm, K. 2001, ApJ, 554, 424

Khomenko, E., Collados, M., \& Felipe, T. 2008, Sol. Phys., 251, 589

Kontogiannis, I., Tsiropoula, G., \& Tziotziou, K. 2010a, A\&A, 510, 41

Kontogiannis, I., Tsiropoula, G., Tziotziou, K., \& Georgoulis, M. K. 2010b, A\&A, 524, A12 (Paper I)

Krijger, J. M., Rutten, R. J., Lites, B. W., et al. 2001, A\&A, 379, 1052

Lites, B. W., Chipman, E. G., \& White, O. R. 1982, ApJ, 253, 367

Lites, B. W., Rutten, R. J., \& Kalkofen, W. 1993, ApJ, 414, 345

Lites, B. W., Kubo, M., Socas-Navarro, H., et al. 2008, ApJ, 672, 1237

Longcope, D., Parnell, C., \& DeForest, C. 2009, ASPC, 415, 178

McIntosh, S. W., \& Judge, P. G. 2001, ApJ, 561, 420

McIntosh, S. W., Fleck, B., \& Judge, P. G. 2003, A\&A, 405, 769

Moon, Y.-J., Kim, Y.-H., Park, Y.-D., et al. 2007, PASJ, 59, S625

Muglach, K. 2003, A\&A, 401, 685

Muglach, K., Hofmann, A., \& Staude, J. 2005, A\&A, 437, 1055

Orozco Suárez, D., Bellot Rubio, L. R., Del Toro Iniesta, J. C., et al. 2007, PASJ, 59,837

Orozco Suárez, D., Bellot Rubio, L. R., Vögler, A., et al. 2010, A\&A, 518, A2

Pérez-Suárez, D., Maclean, R. C., Doyle, J. G., et al. 2008, A\&A, 492, 575

Rosenthal, C. S., Bogdan, T. J., Carlsson, M., et al. 2002, ApJ, 564, 508

Scherrer, P. H., Bogart, R. S., Bush, R. I., et al. 1995, Sol. Phys., 162, 129

Schmidt, H. U. 1964, in Proc. AAS-NASA Symp. on the Physics of Solar Flares, ed. W. N. Hess (Washington DC: NASA), 107

Shchukina, N., \& Trujillo-Bueno, J. 2001, ApJ, 550, 970

Thomas, J. H., \& Stanchfield, D. C. H. 2000, ApJ, 537, 1086

Ulrich, R. K., Bertello, L., Boyden, J. E., et al. 2009, Sol. Phys., 255, 53

Vecchio, A., Cauzzi, G., Reardon, K. P., et al. 2007, A\&A, 461, L1

Vecchio, A., Cauzzi, G., \& Reardon, K. P. 2009, A\&A, 494, 269

Vernazza, J. E., Avrett, E. H., \& Loeser, R. 1981, ApJS, 45, 635

Wang, D., Zhang, M., Li, H., et al. 2009, Sol. Phys., 260, 233

Westendorp Plaza, C., del Toro Iniesta, J. C., Ruiz Cobo, B., et al. 1998, ApJ, 494, 453

Woodard, M. F., \& Chae, J. 1999, Sol. Phys., 184, 239

Zhao, M., Wang, J.-X., Jin, C.-L., et al. 2009, RAA, 9,933 